\title{
A Universal Specification for Multicore Fiber Crosstalk
}

\author{
Joan M. Gené and Peter J. Winzer, Fellow, IEEE
}

\begin{abstract}
In order to transition multi-core fiber (MCF) technologies from their research state to volume production, key design specifications have to be broadly agreed upon, first and foremost an acceptable level of inter-core crosstalk per unit length. Against common belief, we show that MCF crosstalk requirements per unit length are fairly independent of transmission distance in the context of modern coherent optical communication systems. As a consequence, a single value for the tolerable inter-core crosstalk per unit length can be used to specify MCFs, valid from metropolitan $(\sim 100 \mathrm{~km})$ to trans-pacific $(\sim 10,000 \mathrm{~km})$ deployment scenarios. The notion of a universal inter-core crosstalk specification allows for application-independent MCF designs (including a distance-independent optimum core density) and will facilitate the standardization and volume manufacturing of MCF.
\end{abstract}

Index Terms - Coherent communications, fiber capacity, intercore crosstalk, multi-core fibers, nonlinear interference noise.

\section{INTRODUCTION}

$\mathrm{S}$ PACE -division multiplexing (SDM) is considered the only option to overcome the optical network "capacity crunch" [1]. Among the many proposed SDM-specific transmission fibers, low-crosstalk (XT) single-mode multicore fiber (MCF) [2] is likely to be introduced into the network first, as it allows for traditional transmission system architectures without the need for mode-selective optics or multiple-inputmultiple-output (MIMO) digital signal processing. Several recent papers have attempted to assess the impact of XT on MCF system capacity and reach [3]-[6]. However, the resulting XT specifications vary widely with modulation format and transmission reach, leading to the commonly established belief that different network applications, from short-reach to ultra-long-haul, should require different MCF designs in terms of XT per unit length, and as a result in terms of MCF core densities [7]. Designing individual MCFs for different network applications is highly undesirable, though, as it prevents standardization and volume production, which are both important to getting a new technology such as MCF widely

This work was partially supported by the Spanish Ministry of Science, Innovation and Universities under the Talent Promotion Program and the Knowledge Generation Program (ALLIANCE project TEC2017-90034-C2-2R) inside the National Plan for Research and Innovation.

Joan M. Gené and Peter J. Winzer are with Nokia Bell Labs, Holmdel, NJ, USA (e-mail: joan.gene, peter.winzer@nokia-bell-labs.com).

Joan M. Gené is on leave from Universitat Politècnica de Catalunya, Barcelona, Spain (email: joan.gene@upc.edu).

Copyright (c) 2019 IEEE. Personal use of this material is permitted. However, permission to use this material for any other purposes must be obtained from the IEEE by sending a request to pubs-permissions@ieee.org. deployed in commercial networks. It has been loosely conjectured [8] that a universal XT specification per unit length may exist, resulting in a XT requirement of around $-55 \mathrm{~dB} / \mathrm{km}$, independent of the targeted system application. In this paper, we rigorously examine and validate this conjecture. Based on the fact that any amount of XT, no matter how small, will degrade system performance, any meaningful performance metric must include the performance penalty that one is willing to accept in an MCF system relative to a XT-free reference system (i.e., a system using a fiber bundle instead of a MCF). We consequently introduce the notion of a capacity penalty and a reach penalty and assess the tolerable XT for those two. We also determine a capacity-optimum MCF core density, consistent with a distance-independent crosstalk specification.

\section{SYSTEM MODEL}

The rate-reach trade-off of a transmission system using the latest generation of optical transponders that can finely adapt their modulation format to the respective channel conditions through techniques such as probabilistic constellation shaping (PCS) [9],[10], is accurately represented for Nyquist pulses by

$$
S E=2 \cdot \log _{2}\left(1+\frac{1}{\eta_{T R X}} \frac{\overbrace{\left(\eta_{L} P_{A S E}+\chi P_{S}^{3}+\kappa P_{S}\right)}^{S N R}}{P_{S}}\right)
$$

per polarization-multiplexed spatial path, where $P_{S}$ denotes the per-channel (dual-polarization) signal launch power and $P_{A S E}$ is the amplified spontaneous emission (ASE) power within the signal channel's bandwidth. Starting with $P_{A S E}$ as the ASE from ideal distributed amplification [11], the factor $\eta_{L} \geq 1$ captures noise enhancement due to various line system imperfections such as non-ideal amplification. Nonlinear interference noise (NLIN) is represented by the parameter $\chi$, calculated using the formalisms of [12], [13], and depending on many system parameters. (A robustness analysis shows that our final results depend only mildly on these parameters, cf. Sec IV.4.) The aggregate average XT power due to other signals copropagating at the same wavelength in different cores of the MCF is given by $\kappa P_{S}$, but expressing $\mathrm{XT}$ in multiples of $P_{S}$ does not imply equal signal powers per core. In the low coupling regime considered here, $\mathrm{XT}$ can be modeled as additive white Gaussian noise (AWGN) [14], [15], $\kappa$ increases linearly with distance [2], and interactions of XT and fiber nonlinearities can be neglected. As is customary with MCF XT specifications, " $\mathrm{dB} / \mathrm{km}$ " numbers refer to the XT for $1 \mathrm{~km}$ of fiber, to be scaled linearly (not in $\mathrm{dB}$ ) with distance. While some recent work 
suggests significant statistical variations of XT to necessitate an outage treatment [3], the proportionality of average and outage XT values will not impact the general conclusions of this paper. Transponder implementation penalties are captured by $\eta_{T R X} \geq 1$. Maximizing (1) with respect to the signal power $P_{S}$ reveals that the optimum launch power is independent of $X T$,

$$
P_{S, O P T}=\left(\frac{\eta_{L} P_{A S E}}{2 \chi}\right)^{1 / 3},
$$

and the resulting maximum signal-to-noise ratio $(\mathrm{SNR})$ is

$$
S N R_{M A X}=\left(3\left(\frac{\chi \cdot \eta_{L}^{2} P_{A S E}^{2}}{4}\right)^{1 / 3}+\kappa\right)^{-1} .
$$

This interesting fact, which has also been observed experimentally [5],[6], is illustrated in Fig. 1. All results assume MCF with standard single-mode cores $(\alpha=0.2 \mathrm{~dB} / \mathrm{km}, D=$ $17 \mathrm{ps} /(\mathrm{nm} \cdot \mathrm{km}), \gamma=1.31 /(W \cdot \mathrm{km}))$, ideal distributed amplification, 100 wavelength- and polarization-division multiplexed 50-GBaud channels at 50-GHz spacing, ideal Nyquist spectra, and ideal Gaussian constellations, closely approached by, e.g., PCS. Note from Fig. 1 that while the optimum launch power is independent of XT, a flatter optimum is achieved with increasing XT.

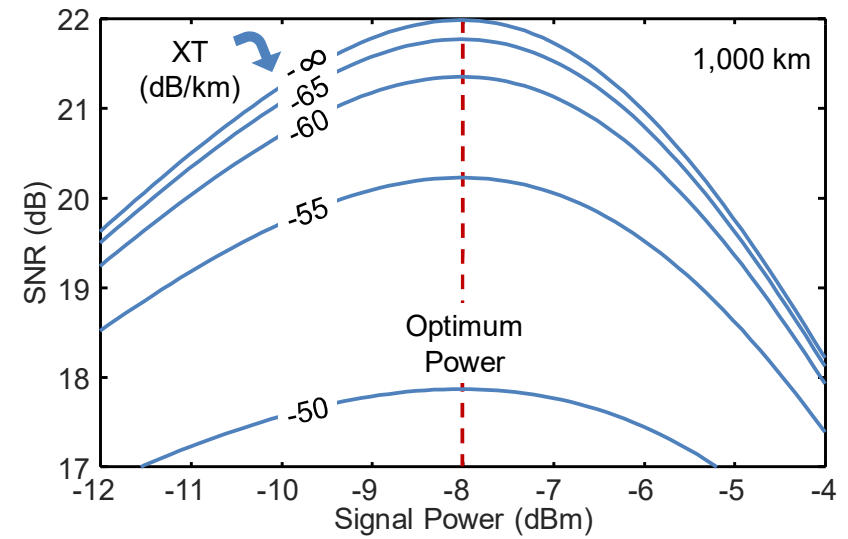

Fig. 1. SNR including ASE, NLIN, and XT at 1,000 km vs. signal launch power with the aggregate XT per kilometer as a parameter.

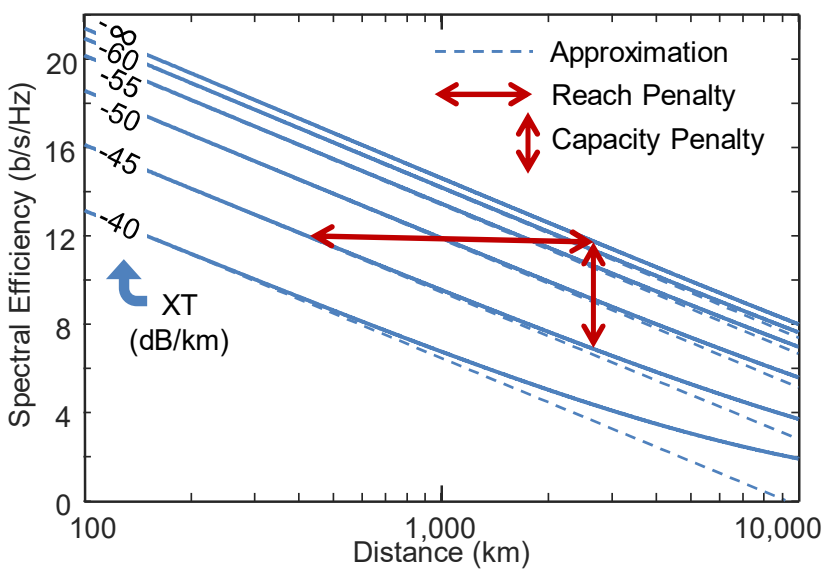

Fig. 2. Spectral efficiency vs. transmission distance for different XT values.

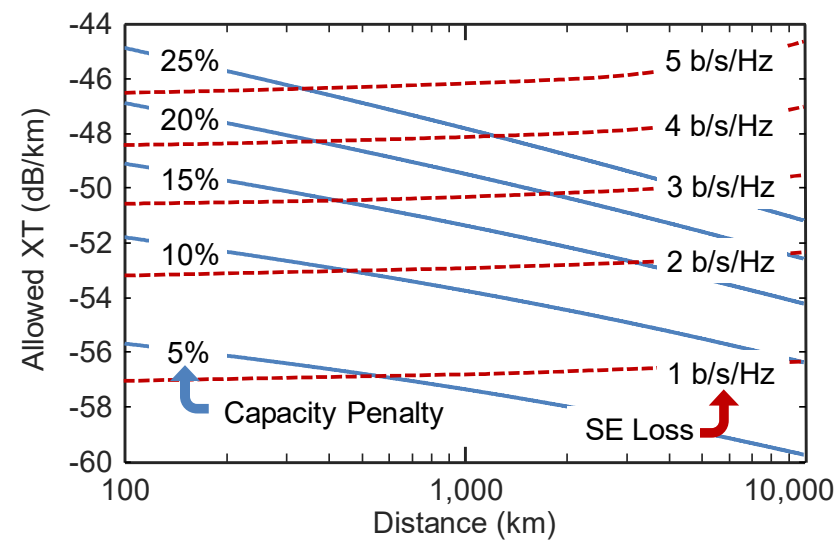

Fig. 3. Maximum allowed XT per kilometer for a given capacity penalty (solid blue lines) and absolute SE loss (dashed red lines).

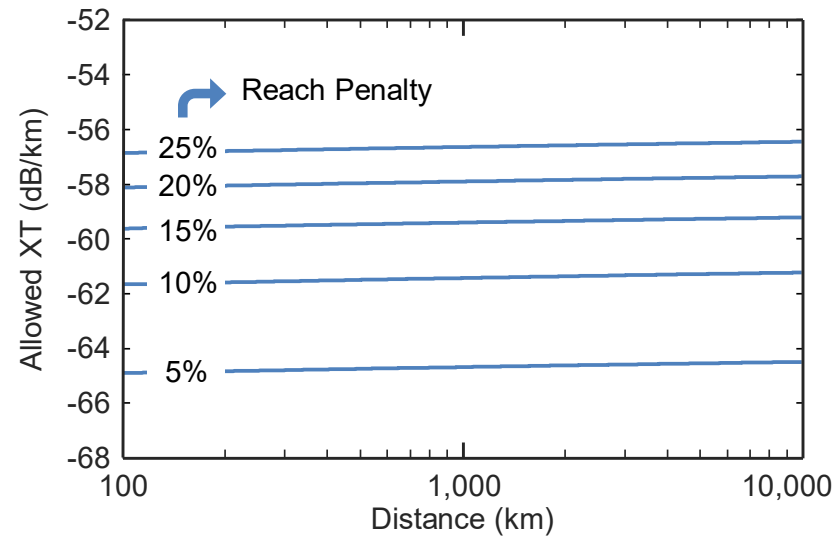

Fig. 4. Maximum allowed XT per kilometer for a given reach penalty.

\section{Two Key MCF System PERFORMANCE METRICS}

Fig. 2 shows the influence of XT on the achievable (dualpolarization) $\mathrm{SE}$ as a function of transmission distance, assuming at each operating point optimized launch powers according to (2) and no implementation penalties, i.e., $\eta_{L}=$ $\eta_{T R X}=1$. (These will be included in Sec. IV.) As ASE, NLIN, and XT are either exactly or to a very good approximation proportional to the transmission distance $L$ [2],[11]-[13], we observe parallel straight lines in the high-SNR regime $(S N R \gg$ 1) at shorter distances and low XT; dashed lines are calculated using Eq. (1) without the " $1+$ " term inside the logarithm for comparison. Two performance metrics become evident:

(i) a reach penalty at a fixed $\mathrm{SE}$, i.e., the ratio $L_{0} / L_{X T}$ (expressed in $\mathrm{dB}$ ) of the maximum transmission distance without $\mathrm{XT}$ to the maximum transmission distance with $\mathrm{XT}$ at a given $\mathrm{SE}$, or in percent $\left(L_{0}-L_{X T}\right) / L_{0}$, and

(ii) an SE penalty at a fixed reach (a capacity penalty when multiplied by the system bandwidth and the number of parallel spatial paths), i.e., the ratio $S E_{0} / S E_{X T}$ (expressed in $\mathrm{dB}$ ) of the achievable SE without XT to the achievable SE with XT at a given reach, or in percent $\left(S E_{0}-S E_{X T}\right) / S E_{0}$.

Note from the logarithmic $\mathrm{x}$-axis scale that the distance penalty $L_{0} / L_{X T}$ (horizontal double-arrow) is approximately constant with transmission reach (exactly for $S N R \gg 1$ ). On the 
other hand, due to the linear y-axis scale, it is the absolute $S E$ difference $S E_{0}-S E_{X T}$ (vertical double-arrow), corresponding to an absolute capacity loss when multiplied with the system bandwidth and the number of parallel spatial paths that is approximately constant with reach (exactly for $S N R \gg 1$ ). This implies an increasing capacity penalty (relative to the XT-free capacity) with transmission distance. Depending on the application scenario, either metric can be important.

\section{TOLERABLE CRosstalK AND ITS UniVERSAlity}

\section{Capacity penalty}

From Fig. 2 we extract the maximum allowed XT per unit length $\hat{\kappa}=\kappa / L$ for a given capacity penalty; the results are shown in Fig. 3 for capacity penalties between $5 \%$ and $25 \%$. As expected, the tolerable XT per unit length decreases only very slightly with transmission reach. For instance, allowing a $10 \%$ capacity penalty for a MCF system relative to a system without any XT lets $\hat{\kappa}$ decrease by less than $5 \mathrm{~dB}$ between $100 \mathrm{~km}$ and $10,000 \mathrm{~km}$. The less XT penalty one accepts, the smaller becomes the variation of the allowable XT with transmission reach. For reference purposes, the maximum allowed XT for a given absolute SE loss is also shown in Fig. 3. As expected, the tolerable XT is nearly constant with distance in this case.

\section{Reach penalty}

Fig. 4 shows the tolerable XT per kilometer with the reach penalty as a parameter. As expected, the required XT per unit length is almost independent of transmission distance for a given reach penalty. Allowing a $10 \%$ reach penalty due to XT, we find the tolerable XT per kilometer to vary by $0.5 \mathrm{~dB}$ between $100 \mathrm{~km}$ and $10,000 \mathrm{~km}$.

\section{Universality of the tolerable $X T$}

Fig.5a summarizes the above results, showing reach penalty (solid-blue) and $S E$ penalty (or capacity penalty, dashed-red) as a function of XT per kilometer for 100,1,000 and 10,000 km. As expected from Fig. 4, the three curves for the reach penalty are practically on top of each other, which corroborates the conjecture that the tolerable XT per kilometer in an MCF system is independent of system reach. On the other hand, specifying an MCF system in terms of its capacity penalty relative to a system using parallel fiber bundles yields a slightly distance-dependent XT specification, as discussed along with Fig. 3.

\section{Robustness to parameter variations}

Implementation penalties $\left(\eta_{L}, \eta_{T R X}\right)$ have a relatively small impact on the tolerable XT, as investigated in Fig. 5b and summarized in Tab. I. When only a transponder implementation penalty $\left(\eta_{T R X}\right)$ is assumed, the reach penalty curves remain unaffected while the capacity penalty curves move slightly towards the reach penalty curves (more restrictive XT requirements). Assuming only a link implementation penalty $\left(\eta_{L}\right)$ slightly relaxes XT requirements for both capacity and reach penalties (both move slightly to the right). Fig. $5 \mathrm{~b}$ corresponds to $\eta_{T R X}=\eta_{L}=3 d B$, letting the overall XT requirements be a little closer together and be slightly displaced to the right (less restrictive XT requirements). This analysis also shows that reasonable changes of $\chi$ will not have a significant effect on XT specifications, as these can be recast in terms of $\eta_{L}$ and $\eta_{T R X}$, cf Eq (1).
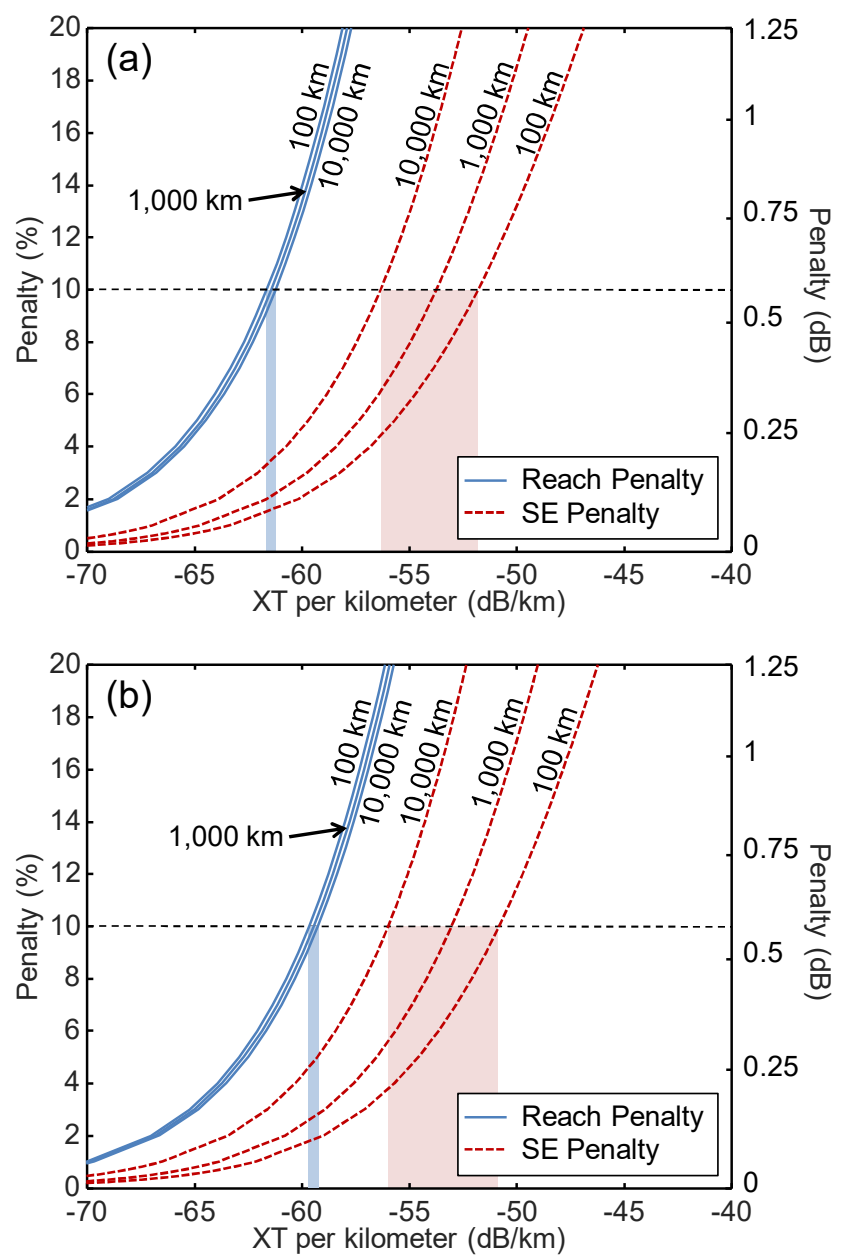

Fig. 5. Reach penalty (solid-blue) and SE penalty (or capacity penalty, dashed-red) vs XT per kilometer for 3 different transmission distances: 100, 1,000 and $10,000 \mathrm{~km}$. (a) No implementation penalties; (b) 3-dB transponder implementation penalty and $3-\mathrm{dB}$ link implementation penalty.

TABLE I

CROSSTALK REQUiREMENTS SUMMARY

\begin{tabular}{|c|c|c|c|c|c|c|c|c|c|}
\hline & \multirow{2}{*}{\multicolumn{2}{|c|}{$\begin{array}{r}\text { Implementation } \\
\text { Penalty } \\
\end{array}$}} & $\eta_{\mathrm{L}}$ & $(\mathrm{dB})$ & 0 & 0 & 3 & 3 \\
\hline & & & & $\eta_{\mathrm{TRX}}$ & $(\mathrm{dB})$ & 0 & 3 & 0 & 3 \\
\hline \multirow{12}{*}{ 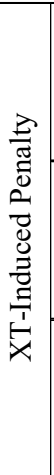 } & \multirow{4}{*}{5} & \multirow{2}{*}{ Reach } & \multirow{12}{*}{ 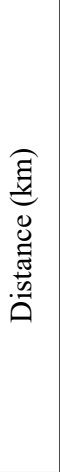 } & 100 & \multirow{12}{*}{ 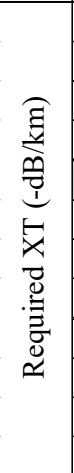 } & 65 & 65 & 62.9 & 62.9 \\
\hline & & & & 10,000 & & 64.5 & 64.5 & 62.4 & 62.4 \\
\hline & & \multirow{2}{*}{ Capacity } & & 100 & & 55.6 & 56.1 & 54 & 54.5 \\
\hline & & & & 10,000 & & 59.6 & 60.7 & 58.3 & 59.3 \\
\hline & \multirow{4}{*}{$10 \%$} & \multirow{2}{*}{ Reach } & & 100 & & 61.7 & 61.7 & 59.7 & 59.7 \\
\hline & & & & 10,000 & & 61.2 & 61.2 & 59.2 & 59.2 \\
\hline & & \multirow{2}{*}{ Capacity } & & 100 & & 51.7 & 52.3 & 50.2 & 50.8 \\
\hline & & & & 10,000 & & 56.3 & 57.3 & 55 & 56 \\
\hline & \multirow{4}{*}{$20 \%$} & \multirow{2}{*}{ Reach } & & 100 & & 58.2 & 58.2 & 56.2 & 56.2 \\
\hline & & & & 10,000 & & 57.7 & 57.7 & 55.7 & 55.7 \\
\hline & & \multirow{2}{*}{ Capacity } & & 100 & & 46.9 & 47.7 & 45.4 & 46.2 \\
\hline & & & & 10,000 & & 52.6 & 53.7 & 51.3 & 52.3 \\
\hline
\end{tabular}


Table I summarizes the results for a $5 \%, 10 \%$ and $20 \%$ penalty in reach and in capacity. For a $10 \%$ reach penalty, the XT specification varies between -59.2 and $-61.7 \mathrm{~dB}$, and for a $10 \%$ capacity penalty, the variation is between $-50.2 \mathrm{~dB}$ and $-57.3 \mathrm{~dB}$, across systems reaching from $100 \mathrm{~km}$ to $10,000 \mathrm{~km}$. Taking the strictest of these values (shown in bold print in Tab. I), we find that an inter-core XT of around $-60 \mathrm{~dB} / \mathrm{km}$ represents a universal XT specification for all considered systems and for both considered penalty metrics at a reasonable penalty level.

\section{OPTIMUM MCF CORE DENSITY}

A related question for MCF standardization is to determine the capacity-optimum MCF core density and whether this design parameter is also distance-independent. While the exact core density will depend on parameters such as core design, cladding diameter, and core lattice, we show here by way of example that the optimum core density can indeed be distance independent to first order, in agreement with our above results.

We use circle packing theory to determine a tightly-packed core lattice [7] for a given cladding diameter and other physical parameters, limited by mechanical constraints [16]. Specifically, we assume a cladding diameter of $260 \mu \mathrm{m}$ and an outer core thickness of $30 \mu \mathrm{m}$. Core-to-core XT decreases exponentially with core-to-core separation [2]; we assume -70 $\mathrm{dB} / \mathrm{km}$ at $1550 \mathrm{~nm}$ for a core-to-core distance of $45 \mu \mathrm{m}$ [17], changing by $3.2 \mathrm{~dB}$ per $\mu \mathrm{m}$ variation of core separation [2]. The other system parameters are the same as in previous sections (with $\eta_{T R X}=\eta_{L}=1$ ). The aggregate MCF capacity under these constraints is shown in Fig. 6, which we found to provide a capacity very close to numerically further optimized values.

The most relevant outcome of Fig. 6 is that the capacityoptimum number of cores for a given core design and fiber outer diameter is nearly independent of transmission distance (varying from 25 at $10,000 \mathrm{~km}$ to 27 at $100 \mathrm{~km}$ ). The absolute loss of capacity (compared to the zero XT case; dashed lines) ranges from 0.5 to $3 \mathrm{~b} / \mathrm{s} / \mathrm{Hz}$ per core, and the XT at the capacityoptimum core density varies from -60 to $-52 \mathrm{~dB} / \mathrm{km}$, consistent with our above analysis, cf. Fig. $3 \mathrm{~b}$.

\section{CONCLUSION}

In view of the results obtained here, we conclude that there exists a universal target for the XT per unit length of MCF, around $-60 \mathrm{~dB} / \mathrm{km}$, which only mildly depends on the penalty definition, the penalty level, and the network scenario in which the fiber is to be deployed. Specifying such universally applicable XT values is expected to help the MCF industry to standardize and develop widely deployable MCFs with the required volume to warrant the commercialization of this emerging technology.

\section{ACKNOWLEDGMENT}

The authors gratefully acknowledge valuable discussions with Takashi Sasaki, Tetsuya Hayashi, Ronen Dar, Ruben S. Luís, Ben J. Puttnam, and Werner Klaus.

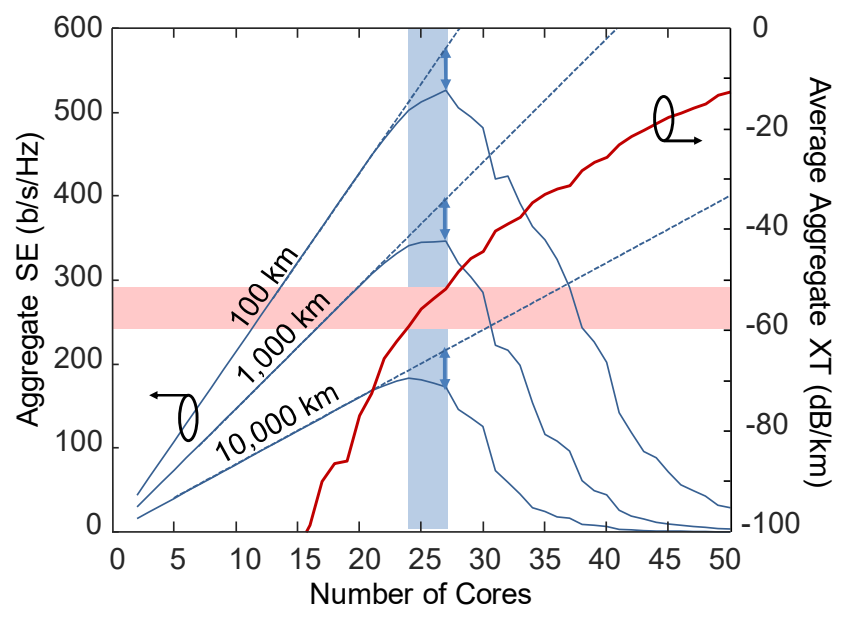

Fig. 6. Aggregate spectral efficiency (left axis) and aggregate XT (right axis) as a function of the number of cores.

\section{REFERENCES}

[1] P. J. Winzer et al., "Fiber-optic transmission and networking: the previous 20 and the next 20 years," Opt. Express, p. 24190, 2018.

[2] K. Saitoh and S. Matsuo, "Multicore Fiber Technology," J. Lightwave Technology, pp. 55-66, Jan. 2016.

[3] B. J. Puttnam et al., "Impact of Intercore Crosstalk on the Transmission Distance of QAM Formats in Multicore Fibers," IEEE Photonics Journal, pp. 1-9, Apr. 2016.

[4] R. S. Luís et al., "Long distance crosstalk-supported transmission using homogeneous multicore fibers and SDM-MIMO demultiplexing," Opt. Express, p. 24044, 2018.

[5] R. S. Luís et al., "Spectral efficiency in crosstalk-impaired multi-core fiber links," in Optical Data Science: Trends Shaping the Future of Photonics, vol. 10551, p. 1055102, 2018.

[6] D. J. Elson et al., "Impact of Intercore Crosstalk on Achievable Information Rates," IEEE Photonics Summer Topicals Meeting, pp. 2122, 2018.

[7] R. S. Luís et al., "On the Spectral Efficiency Limits of Crosstalk-Limited Homogeneous Single-Mode Multi-Core Fiber Systems," in Advanced Photonics 2017, p. NeTu2B.2, 2017.

[8] P. J. Winzer, "From first fibers to mode-division multiplexing," Chinese Opt. Letters, pp. 10-14, 2016.

[9] F. Buchali et al., "Rate Adaptation and Reach Increase by Probabilistically Shaped 64-QAM: An Experimental Demonstration," J. Lightwave Technology, pp. 1599-1609, Apr. 2016.

[10] J. Cho et al., "Trans-Atlantic Field Trial Using High Spectral Efficiency Probabilistically Shaped 64-QAM and Single-Carrier Real-Time 250Gb/s 16-QAM," J. Lightwave Technology, pp. 103-113, Jan. 2018.

[11] R.-J. Essiambre et al., "Capacity Limits of Optical Fiber Networks," J. Lightwave Technology, pp. 662-701, Feb. 2010.

[12] P. Poggiolini et al., "The GN-Model of Fiber Non-Linear Propagation and its Applications," J. Lightwave Technology, pp. 694-721, Feb. 2014.

[13] R. Dar et al., "Accumulation of nonlinear interference noise in fiber-optic systems,” Opt. Express, p. 14199, Jun. 2014.

[14] T. Hayashi et al., "Behavior of inter-core crosstalk as a noise and its effect on Q-factor in multi-core fiber," IEICE Transactions on Communications, pp. 936-944, 2014.

[15] T. Hayashi et al., "Uncoupled multi-core fiber enhancing signal-to-noise ratio," Opt. Express, p. B94, 2012.

[16] S. Matsuo et al., "High-Spatial-Multiplicity Multicore Fibers for Future Dense Space-Division-Multiplexing Systems," J. Lightwave Technology, pp. 1464-1475, 2016.

[17] B. J. Puttnam et al., "High capacity multi-core fiber systems," in 21 st European Conference on Networks and Optical Communications (NOC), pp. 29-34, 2016. 